\title{
ON ONE-PARAMETER SUBGROUPS IN FINITE DIMENSIONAL LOCALLY COMPACT GROUP WITH NO SMALL SUBGROUPS
}

\author{
MASATAKE KURANISHI
}

Let $G$ be a locally compact topological group and let $U$ be a neighborhood of the identity in $G$. A curve $g(\lambda)(|\lambda| \leqq 1)$ in $G$, which satisfies the conditions,

$$
g(s) g(t)=g(s+t) \quad(|s|,|t|,|s+t| \leqq 1),
$$

is called a one-parameter subgroup of $G$. If there exists a neighborhood $U_{1}$ of the identity in $G$ such that for every element $x$ of $U_{1}$ there exists a unique one-parameter subgroup $g(\lambda)$ which is contained in $U$ and $g(1)=x$, we shall call, for the sake of simplicity, that $U$ has the property $(\mathrm{S})^{*}$. It is well known that the neighborhoods of the identity in a Lie group have the property $(S)$. More generally it is proved that if $G$ is finite dimensional, locally connected, and is without small subgroups, ${ }^{1)} G$ has the same property. ${ }^{2}$ In this note, these theorems will be generalized to the case when $G$ is finite dimensional and without small subgroups.

The writer's proof is based on the theorems recently developed by D. Montgomery and A. Gleason. ${ }^{3}$ Their theorems, which will be used in this note, are summarized in $\S 1$. In $\S 2$ it will be proved that the group $G$, which is finite dimensional and without small subgroups, is locally connected and our theorem is reduced to the known case.

$\S 1$. THEOREM 1 (Montgomery). ${ }^{4)}$ Let $G$ be a locally compact locally connected n-dimensional group $(n<\infty)$. Then there exists a neighborhood $V$ of the identity in $G$ possessing the following properties:

Let $A$ and $B,(B \subset A)$, be compact subsets of $V$. Then the sufficient con-

Received November 5, 1951.

1) $G$ is called to be without small subgroups, if there exists a compact neighborhood of the identity in $G$ which does not contain non-trivial subgroups of $G$.

$\Rightarrow$ Cf. Chevalley, C. [1]. C. Chevalley proved the case when $G$ is locally euclidean and without small subgroups. K. Iwasawa communicated to the present author that D. Montgomery pointed out that the Chevalley's method may be applicable even when $G$ is locally connected and without small subgroups. It is also informed that H. Yamabe obtained the same result.

3) D. Montgomery [7], [8], [9], [10], A. Gleason [2].

4) This Theorem and its Corollaries are valid when $G$ is a locally connected finite dimensional homogeneous space, or more generally, $G$ is a locally homogeneous space. See D. Montgomery [8]. 
ditions for $A-B$ to be an open subset of $G$ are

1) $B$ carries an $(n-1)$-cycle $z^{5)}$ which is not homologous to zero in $B$, and

2) $A$ is minimal with respect to the properties

a) $B \subset A$

b) $z$ is homologous to zero in $A$.

Corollary 1 to Theorem 1. (Invariance theorem of domain). Let $G_{1}$ and $G_{2}$ be locally compact locally connceted groups. Suppose that $\operatorname{dim} G_{1}=\operatorname{dim} G_{2}$ $=n<\infty$. Let $M$ be an open subset of $G_{1}$ and $f$ be a topological mapping of $M$ into $G_{2}$. Then the image $f(M)$ of $M$ under the mapping $f$ is an open subset of $G_{2}$.

Proof. Let $V_{i}$ be the neighborhood of the identity in $G_{i}$ pointed out in Theorom $1(i=1,2)$. Let $p_{2}$ be a point of $f(M)$ and let $p_{1}$ be the ponit $M$ such that $f\left(p_{1}\right)=p_{2}$. We can take a neighborhood $V_{1}^{\prime}$ of the identity in $G_{1}$ such that $\bar{V}_{1}^{\prime} \leqq V_{1}, \bar{V}_{1}^{\prime} p_{1} \leqq M, f\left(\bar{V}_{1}^{\prime} p_{1}\right) \subseteq V_{2} p_{2}$. Since the dimension of $\bar{V}_{1}^{\prime}$ is $n$, there exist compact subsets $A_{1}$ and $B_{1}$ of $\bar{V}_{1}^{\prime}$ satisfying the conditions 1) and 2) of the Theorem 1. ${ }^{6}$ Moreover, we can assume that the identity in $G_{1}$ is contained in $A_{1}-B_{1}$. Then $f\left(A_{1} p_{1}\right)$ and $f\left(B_{1} p_{1}\right)$ are subsets of $V_{2} p_{2}$ and satisfy the conditions 1) and 2) of the Theorem 1 . Hence by Theorem $1 f\left(A_{1} p_{1}\right)-f\left(B_{1} p_{1}\right)$ is an open subset of $G_{2}$. Since $p_{2} \in f\left(A_{1} p_{1}\right)-f\left(B_{1} p_{1}\right) \subseteq f(M), f(M)$ is an open subset of $G_{2}$.

Corollary 2 to Theorem 1. Under the same notations and assumptions as in the Corollary 1 , let $N$ be an open subset of $M$ such that $\bar{N} \cong M$, and let $x$ be an arbitrary point of $f(N)$. Then

$$
C_{x}\left(G_{2}-f(b d r y N)\right)^{7)} \leqq f(N) .
$$

Proof. From the Corollary 1, it is easy to prove that

$$
\operatorname{bdry} f(N)=f(b d r y N) .
$$

Hence, $G_{2}-f(b d r y N)=G_{2}-b d r y f(N)=f(N) \cup\left(G_{2}-\overline{f(N)}\right)$, $f(N) \cap\left(G_{2}-\overline{f(N)}\right)=\phi$, , and both $f(N)$ and $\left(G_{2}-\overline{f(N)}\right)$ are open subsets of $G_{2}$. Since $x \in f(N)$, it follows that

$$
C_{x}\left(G_{2}-f(b d r y N) \leqq f(N)\right) .
$$

THEOREM $2^{9)}$ (Montgomery). Let $G$ be a locally compact n-dimensional

5) Cycles are in the sense of Cech.

6) Cf. Hurewicz and Wallman [2], p. 151.

7) If $x$ is a point of topological space $A, C_{x}(A)$ is the connected component of $A$ which contains $x$.

8) $\phi$ denotes the empty set.

9) This is a part of Theorem 7 of D. Montgomery [9]. 
group $(n<\infty)$. Then there exists a locally compact locally connected group $G^{*}$ of dimension $n$ and a continuous one-to-one mapping $\alpha$ of $G^{*}$ into $G$ satisfying the following conditions.

$\left\{\begin{array}{l}\text { Let } C^{*} \text { be a neighborhood of the identity in } G^{*} \text {, then } \alpha\left(C^{*}\right)=C \text { is an } \\ \text { invariant local snbgroup of } G \text { and the factor local group of } G \text { by } C \text { is zero- } \\ \text { dimensional. }\end{array}\right.$

§2. A neighborhood $U$ of the identity in a topological group $G$ is called to have the property $(S)$, if for every element $x$ of $U$ there exists an integer $n$ such that $x^{2 /} \notin U$.

LeMma 1 (Yamabe). ${ }^{10)}$ Let $G$ be a locally compact group, and suppose that $G$ is without small subgroups. Let $U$ be a neighborhood of the identity $e$ in $G$ such that $U$ contains no non-trivial subgroups. For every neighborhood $V$ of $e$ there exists a neighborhood $V^{*}$ of $e$ satisfying the following conditions.

If $x$ and $x^{k}$ are contained in $V^{*}$ and if $x^{i}(1 \leqq i \leqq k)$ are elements of $U$, then $x^{i}$ is contained in $V$ for $i=1,2, \ldots, k$.

Corollary to Lemma 1 (Yamabe and Gotô). ${ }^{11)}$ If a locally compact group $G$ is without small subgroups, $G$ has the property (S).

LEMMA $2 .^{12)}$ Let $G$ be a locallv compact group which is without small subgroups. Then there exists a neighborhood $U$ of the identity in $G$, in which the square root is unique. More strictly, if $x$ and $y$ are elements of $U$, and if $x^{2}$ $=y^{2}$, it follows that $x=y$.

In this case the mapping $\varphi(x)=x^{2}$ of $U$ into $G$ is one-to-one.

LEMMA $3^{12)}$ Let $G$ be a locally compact group which is without small subgroups. Then on a sufficiently small neighborhood $U$ of the identity in $G$ we can define a real valued continuous function $f(x)$ satisfying the following conditions.

$$
\begin{gathered}
f\left(x^{2}\right) \geqq 2 f(x) \text { for } x, x^{2} \in U, \\
f(x)=0 \text { if and only if } x \text { is the identity. }
\end{gathered}
$$

Now let $U$ be a local group and let $C$ be an invariant local subgroup of $U$. If we take a sufficiently small neighborhood $W$ of the identity in $U$ the factor local group $W / C$ is defined as follows. ${ }^{13)}$

(i) The element $X$ of $W / C$ is the coset $W \cap C x$ for $x \in W$.

(ii) We shall consider that the product $X Y$ of a pair of elements $X, Y$ of $W / C$ is defined if and only if there exist elements $x \in X$ and $y \in Y$ such that

\footnotetext{
10) For the proof, see H. Yamabe [12].

11) H. Yamabe and M. Gotô [4].

12) See Kuranishi [5] and [6].

13) Pontrjagin [11], p. 83.
} 
$x y$ is contained in $W$. The product $X Y$ is equal to $W \cap C x y$, which is independent of the choices of $x$ and $y$.

(iii) The natural mapping $W \rightarrow W / C$ is continuous and open.

Let $G$ be a locally compact finite dimensional group. Suppose that $G$ is without small subgroups. Let $G^{*}$ and $\alpha$ be the locally compact locally connected group and the continuous one-to-one mapping of $G^{*}$ into $G$ stated in Theorem 2. Let $U$ be the sufficiently small neighborhood of the identity in $G$ on which the function $f(x)$ of Lemma 2 is defined. $U$ is naturally a local group. Take a sufficiently small open neighborhood $C^{*}$ of the identity in $G^{*}$ and let $C=\alpha\left(C^{*}\right)$. By Theorem $2 C$ is an invariant local subgroup of $U$. Take a sufficiently small neighborhood $W_{1}$ of the identity in $U$ so that the factor local group $W_{1} / C$ is defined. By Theorem $2 W_{1} / C$ is a zero-dimensional locally compact local group. Let $\beta$ be the natural mapping $W_{1} \rightarrow W_{1} / C$ and let $\varphi$ be a mapping $\varphi(x)=x^{2}$. Take an open neighborhood $W$ of the identity in $U$ such that $\bar{W} \leqq W_{1}$. Let $V_{1}$ be the neighborhood of the identiy in $U$ such that

$$
\begin{gathered}
\varphi(b d r y W) \cap V_{1}^{2}=\phi, \\
V_{1}^{2} \subseteq W, \\
V_{1} \cap C \text { is connected. }
\end{gathered}
$$

Let $V$ be a neighborhood of the identity in $U$ such that $V^{4} \cong V_{1}, V=V^{-1}$.

LEMMA 4. Let $X$ be an element of $\beta(V)$ such that $X^{2}$ is contained in $\beta(V)$. Then for every element $y$ of $X^{2} \cap \bar{V}$, there exists an element $x$ of $X$ such that $y=x^{2}$.

Proof. Let

$$
X=W_{1} \cap C x_{0}, \quad x_{1} \in V,
$$

and

$$
M^{*}=\alpha^{-1}\left(\left(W_{1} \cap C x_{0}\right) x_{0}^{-1}\right) \text {. }
$$

We define the topological mapping $\psi(a)$ of $M^{*}$ into $G^{*}$ by

$$
\phi(a)=\alpha^{-1}\left(\left(\varphi\left((\alpha(a)) x_{0}\right)\right) x_{0}^{-2}\right) .^{14)}
$$

Since $N^{*}=\alpha^{-1}\left(\left(W \cap C x_{0}\right) x_{0}^{-1}\right)$ is an open set containing the identity $e^{*}$ in $G^{*}$ and $\bar{N}^{*} \cong M^{*}$, by Corollars 2 to Theorem 1 ,

$$
C_{e^{*}}\left(G^{*}-\psi\left(b d r y N^{*}\right)\right) \leqq \psi\left(N^{*}\right) \text {. }
$$

Since

$$
\begin{aligned}
& \alpha\left(\alpha^{-1}\left(V_{1} \cap C\right) \cap \psi\left(b d r y N^{*}\right)\right) \\
& \subseteq\left(V_{1} \cap C\right) \cap\left(\varphi\left(b d r y\left(W \cap C x_{0}\right)\right)\right) x_{0}^{-2} \\
& \subseteq\left[V_{1} x_{0}^{2} \cap \varphi(b d r y W)\right] x_{0}^{-2} \\
& \left.\subseteq\left[V_{1}^{2} \cap \varphi(b d r y W)\right] x_{0}^{-2}=\phi \quad \text { (by condition }(5)\right)
\end{aligned}
$$

14 $\alpha$ is the injection of $G^{*}$ into $G$. 
and since $V_{1} \cap C$ is connected, it follows that

$$
\alpha^{-1}\left(V_{1} \cap C\right) \leqq C_{e^{*}}\left(G^{*}-\psi\left(b d r y N^{*}\right)\right) \text {. }
$$

If $c x_{0}^{2} \in X^{2} \cap \bar{V}=\left(W_{1} \cap C x_{0}^{2}\right) \cap \bar{V}=\bar{V} \cap C x_{0}^{2}$, it follows that

$$
c \in \bar{V} x_{0}^{-2} \cap C \leqq \bar{V} V^{-2} \cap C \leqq V_{1} \cup C,
$$

that is,

$$
\begin{gathered}
x^{2} \cap \bar{V} \leqq\left(V_{1} \cap C\right) x_{0}^{2} \\
\alpha^{-1}\left[\left(X^{2} \cap \bar{V}\right) x_{0}^{-2}\right] \leqq \alpha^{-1}\left(V_{1} \cap C\right) .
\end{gathered}
$$

From (8), (9) and (10), it follows that

$$
\alpha^{-1}\left[\left(X^{2} \cap \bar{V}\right) x_{0}^{-2}\right] \subseteq \psi\left(N^{*}\right)=\alpha^{-1}\left[\zeta\left(W \cap C x_{0}\right) x_{0}^{-2}\right],
$$

that is,

$$
X^{2} \cap V \subseteq \varphi\left(W \cap C x_{1}\right)
$$

Hence the lemma is proved.

We now define the function $F(X)$ on $\beta(W)$ by

$$
F(X)=\inf _{x \in X \cap \bar{L}} f(x) .^{15)}
$$

Lemma 5. Let $V$ be the neighborhood of the identity in $G$ stated in Lemma 4. We can assume without loss of generality that $V=\{x \mid f(x) \leqq \delta\}$, where $f(x)$ is the function of Lemma 3. Then

$$
\begin{aligned}
& F\left(X^{2}\right) \geqq 2 F(X) \quad \text { if } X, X^{2} \in \beta(V), \\
& F(X)=0 \text { if and only if } X \text { is the identity, } \\
& F(X) \text { is continuous. }
\end{aligned}
$$

Proof. Continuity of $F(X)$ : Let $X_{n} \in \beta(V)$, and $X_{n} \rightarrow X \in \beta(V)$. There exists a sequence $x_{n}(n=1,2, \ldots)$ of $V$ such that $F\left(X_{n}\right)=f\left(x_{n}\right)$. We can assume without loss of generality that $x_{n} \rightarrow x \in V \cap X$. Then

$$
F(X) \leqq f(x)=\lim _{n \rightarrow \infty} f\left(x_{n}\right)=\lim _{n \rightarrow \infty} F\left(X_{n}\right) .
$$

Let $x$ be the element of $X$ such that $F(X)=f(x)$. For arbitrary positive number $\varepsilon$, there exists a neighborhood $V_{2}$ of the identity in $G$ such that

$$
f(y) \leqq f(x)+\varepsilon \text { for } y \in V_{2} x .
$$

Since $\beta$ is an open mapping, there exists an integer $N^{\prime}$ such that

$$
X_{n} \in \beta\left(V_{2} x\right) \text { for } n>N^{\prime} .
$$

15) $f(x)$ is the function of Lemma 3 . 
Let $x_{n}$ be a point of $X_{n} \cap V_{2} x,\left(n=N^{\prime}+1 . N^{\prime}+2, \ldots\right)$. Then

$$
F\left(X_{n}\right) \leqq f\left(x_{n}\right) \leqq f(x)+\varepsilon=F(X)+\varepsilon \text { for } n \leqq N^{\prime},
$$

from (15) and (16) it follows that $F(X)$ is a continuous function on $\beta(\bar{W})$.

(13) is obvious. We shall prove (12). Suppose that $X$ and $X^{2}$ are elements of $\beta(V)$. There exists an element $y$ of $X^{2} \cap V$ such that

$$
F\left(X^{2}\right)=f(y) \text {. }
$$

From Lemma 4 and the fact that $V=\{x \mid f(x) \leqq \delta\}$, there exists an element $x$ of $X \cap V$ such that $x^{2}=y$.

Hence

$$
F\left(X^{2}\right)=f(y)=f\left(x^{2}\right) \geqslant 2 f(x) \geqslant 2 F(X) .
$$

Leмма 6. Let $G$ be a locally compact finite dimensional group. Suppose that $G$ is witout small subgroups. Then $G$ is locallv connected.

Proof. Let $V$ be a sufficiently small neighborhood of the identity in $G$. Since $W / C$ is a zero-dimensional local group, $\beta(V)$ contains an open and compact subgroup $H$ of $W / C$. We can take $H$ so that $H$ is the group in the large, i.e., the product is defined for every pair of elements of $H$ and is contained in $H{ }^{17}$ By Lemma 5 there is defined the function $F(X)$ on the compact group $H$ and satisfies the conditions

$$
F\left(X^{2}\right) \geqq 2 F(X) \text { for every element } X \text { of } H \text {. }
$$

(13). and (14). Hence $H$ must be the group consisting of the identity element only. Since $H$ is an open subset of $W / C, W / C$ must be a discrete space. Thus $W$ is locally connected.

Theоrem 3. Let $G$ be a finite dimensional locally.compact group. Suppose that $G$ is without small subgroups. Then for every neighborhood $U$ of the identity in $G$ there exists a neighborhood $U_{1}$ satisfying the following conditions.

"For every element $x$ of $U_{1}$, there exists a unique one-parameter subgroup $g(\lambda)(0 \leqq \lambda \leqq 1)$ contained in $U$ such that $g(1)=x . "$

Proof. We can suppose without loss of generality that

(18) the function $f(x)$ of Lemma 3 is defined on $U$, and that

(19) the mapping $\varphi(x)=x^{2}$ of $U$ into $G$ is one-to-one. (Lemma 2.)

Take a neighborhood $V$ of the identity in $G$ such that $V^{2} \leqq U$ and let $V^{*}$ be an open neighborhood of the identity in $G$ of the Lemma 1 with respect to $V$. By Lemrna $6, G$ is locally. connected. Hence from the condition (19) and

15. This can be proved in the same way as in the case of the locally compact zero-dimensional groups. 
the Corollary 1 to Theorem $1, \varphi\left(V^{*}\right)$ is an open subset $G$ and contains the identity. Choose a sufficiently small positive number $\delta$ such that

$$
U_{1}=\{x \mid f(x)<\delta\} \cong V^{*} \cap \varphi\left(V^{*}\right) .
$$

For every element $x$ of $U_{1}$, there exists an element $x_{1}$ of $V^{*}$ such that $x$ $=x_{10}^{2}$ Since $f\left(x_{1}\right) \leqq \frac{1}{2} f\left(x_{1}^{2}\right)=\frac{1}{2} f(x)<\delta, x_{1}$ is contained in $U_{1}$. Thus there exists a suquence $x_{n}(n=1,2, \ldots)$ of elements of $U_{1}$ such that

$$
x=x_{13}^{2 n} \text { 。 }
$$

Since the square root is unique (Lemma 2),

$$
x_{n} x_{m}=x_{n} x_{n}
$$

and

$$
x_{n}=x_{m}^{2^{m-n}} \text { for } \quad m \geqslant n
$$

Then there exists a unique one-parameter subgroup $g(\lambda)$ such that $g\left(\frac{1}{2^{n}}\right)-x_{n}$ for $n=1,2, \ldots{ }^{1 i}$ Suppose that

$$
g\left(\begin{array}{l}
m \\
2^{n}
\end{array}\right) \subseteq V \quad \text { for } \quad m=1,2, \ldots, 2^{n}
$$

Put $y=g\left(\frac{1}{2^{n+1}}\right) \in U_{1}$. For $m=2 m^{\prime}+1$,

$$
y^{\prime \prime}=g\left(\frac{m}{2^{n+1}}\right)=g\left(\frac{m^{\prime}}{2^{n}}\right) g\left(\frac{1}{2^{n+1}}\right) \in V^{2} \cong U .
$$

Hence

$$
y^{m} \in U \quad \text { for } \quad m s=1,2, \ldots, 2^{n+1} \text {, }
$$

and

$$
y, y^{2^{u+1}} \in U_{1} \subseteq V^{*} \text { 。 }
$$

By Lemma 1, $\quad y^{m} \in V$ for $m=1,2, \ldots 2^{n+1}$.

Hence

$$
g\left(\begin{array}{c}
m \\
2^{n}
\end{array}\right) \equiv V \subseteq U \quad \text { for } \quad m=1,2, \ldots, 2^{n}, \quad n=1,2, \ldots
$$

Thus

$$
g(\lambda) \in V \subseteq U \text { for } \quad 0 \leqq \lambda \leqq 1 \text {. }
$$

BIBLIOGRAPHY

[1] Chevalley, C., On a theorem of Gleason, Proc. Amer. Math. Soc. Vol. 2 (1951) p. 122-

37) See the Lemma 1 of M. Kuranishi [6]. 
125.

[2] Crleason, A., Arcs in locally compact groups, Proc. Nat. Acad. Sci. U.S.A., 36 (1950) pp. 663-667.

[ 3 ] Hurewicz, W. and Wallman, H., Dimension theory, Princeton, 1941.

[4] Gotô, M. and Yamabe, H., On some properties of locally compact groups with no small subgroups, Nagoya Math. Jour. Vol. 2 (1950) pp. 29-33.

[5] Kuranishi, M., On euclidean local groups satisfying certain conditions, Proc. Amer. Math. Soc. Vol. 1 (1950) pp. 372-380.

[6] Kuranishi, M., On conditions of differentiability of locally compact groups, Nagoya Math. Jour. Vol. 1 (1950) pp. 71-81.

[7] Montgomery, D., Theorems on the topological structure of locally compact groups, Ann. of Math. Vol. 50 (1949) pp. 570-580.

[8] Montgomery, D., Locally homogeneous spaces, Ann. of Math. Vol. 52 (1950) pp. 261271.

[9] Montgomery, D., Finite dimensional groups, Ann. of Math. Vol. 52 (1950) pp. 591-605.

[10] Montgomery, D., Existence of subgroups isomorphic to the real numbers, Ann. of Math. Vol. 53 (1951) pp. 298-326.

[11] Pontrjagin, L., Topological groups, Princeton, 1939.

[12] Yamabe, H., Note on locally compact groups, Osaka Math. Jour. Vol. 3 (1951) pp. 2933.

Mathematical Institute, Nagoya University 